$01 ; 11$

\title{
Воздействие гармонического сигнала на генератор квазипериодических колебаний Анищенко-Астахова
}

\author{
(C) А.П. Кузнецов, Ю.В. Седова
}

Саратовский фрилиал Института радиотехники и электроники им. В.А. Котельникова РАН, Саратов, Россия

E-mail: sedovayv@yandex.ru

Поступило в Редакцию 16 июня 2021г.

В окончательной редакции 22 ноября 2021 r.

Принято к публикации 29 ноября 2021 г.

Рассматривается гармоническое воздействие на модифицированный генератор Анищенко-Астахова, способный демонстрировать в автономном режиме двухчастотные квазипериодические колебания. Показана возможность удвоения трехчастотных торов в неавтономной системе. Продемонстрирован эффект подавления хаоса внешним сигналом, приводящий не только к периодическим, но и к квазипериодическим режимам при превышении амплитудой воздействия некоторого порога.

Ключевые слова: удвоения тора, подавление хаоса, генератор квазипериодических колебаний.

DOI: 10.21883/PJTF.2022.04.52086.18925

Радиофизический генератор, предложенный Анищенко и Астаховым, может считаться одной из базовых моделей, демонстрирующей детерминированный хаос [1]. Он представляет собой трехмерную динамическую систему и хорошо изучен как теоретически, так и экспериментально (см. монографии [2,3] и ссылки в них). В работе [4] была предложена его модификация, которая обеспечивает не только периодические и хаотические режимы, но и автономные квазипериодические колебания. С этой целью используется колебательный контур в цепи обратной связи, что дает новую дополнительную частоту. В результате получается автономная четырехмерная модель, которая оказывается удобной для исследования квазипериодических колебаний. В [5] были проведены исследования такого генератора и показана возможность эффекта удвоения двухчастотного тора с ростом параметра возбуждения. Для двух связанных генераторов обсуждались проблема синхронизации резонансного предельного цикла на торе, возникновение резонансных двух- и трехчастотных торов на поверхности четырехчастотного тора, влияние шума на четырехчастотный тор и др. [5-7]. Исследовался вопрос о возникновении гиперхаоса в результате вторичной бифуркации Неймарка-Сакера $[8,9]$. В то же время воздействие гармонического сигнала на модифицированный генератор практически не изучалось. Однако эта задача представляется важной с позиций построения достаточно полной картины синхронизации квазипериодических колебаний.

Уравнения модифицированного генератора Анищенко-Астахова имеют вид [4]:

$$
\begin{gathered}
\dot{x}=m x+y-x \varphi-d x^{3}, \\
\dot{y}=-x, \\
\dot{z}=\varphi, \\
\dot{\varphi}=-\gamma \varphi+\gamma \Phi(x)-g z,
\end{gathered}
$$

где

$$
\Phi(x)=I(x) x^{2}, \quad I(x)=\left\{\begin{array}{l}
1, x>0 \\
0, x \leqslant 0
\end{array}\right.
$$

Здесь $m$ - параметр возбуждения генератора, $d-$ параметр нелинейной диссипации, $\gamma$ - параметр затухания, $g$ - параметр инерционности фильтра, обеспечивающего вторую независимую частоту. Будем использовать значения параметров $d=0.001, \gamma=0.2, g=0.5$.

Добавим теперь внешнее гармоническое воздействие:

$$
\begin{gathered}
\dot{x}=m x+y-x \varphi-d x^{3}+a \cos \omega t, \\
\dot{y}=-x, \\
\dot{z}=\varphi, \\
\dot{\varphi}=-\gamma \varphi+\gamma \Phi(x)-g z,
\end{gathered}
$$

где $a$ и $\omega-$ амплитуда и частота воздействия.

В этом случае с ростом параметра возбуждения $m$ может наблюдаться удвоение, но уже не двухчастотного, а трехчастотного тора. Это утверждение иллюстрирует рис. 1. На вставках к этому рисунку показаны портреты аттракторов в двойном сечении Пуанкаре. Поясним технику построения такого сечения. Результатом обычного сечения Пуанкаре для системы, испытывающей внешнее гармоническое воздействие, является набор точек, получаемых в результате стробоскопического сечения. Для построения двойного сечения мы учитывали только те точки из упомянутого набора, которые попадают в некоторый тонкий слой фазового пространства, задаваемый, например, условием $|x| \leqslant 0.005$. Результат двойного сечения (т. е. стробоскопического сечения и сечения плоскостью $x=0$ ) фазового пространства системы (3) представлен на рисунке. В двойном сечении трехчастотный тор выглядит как 


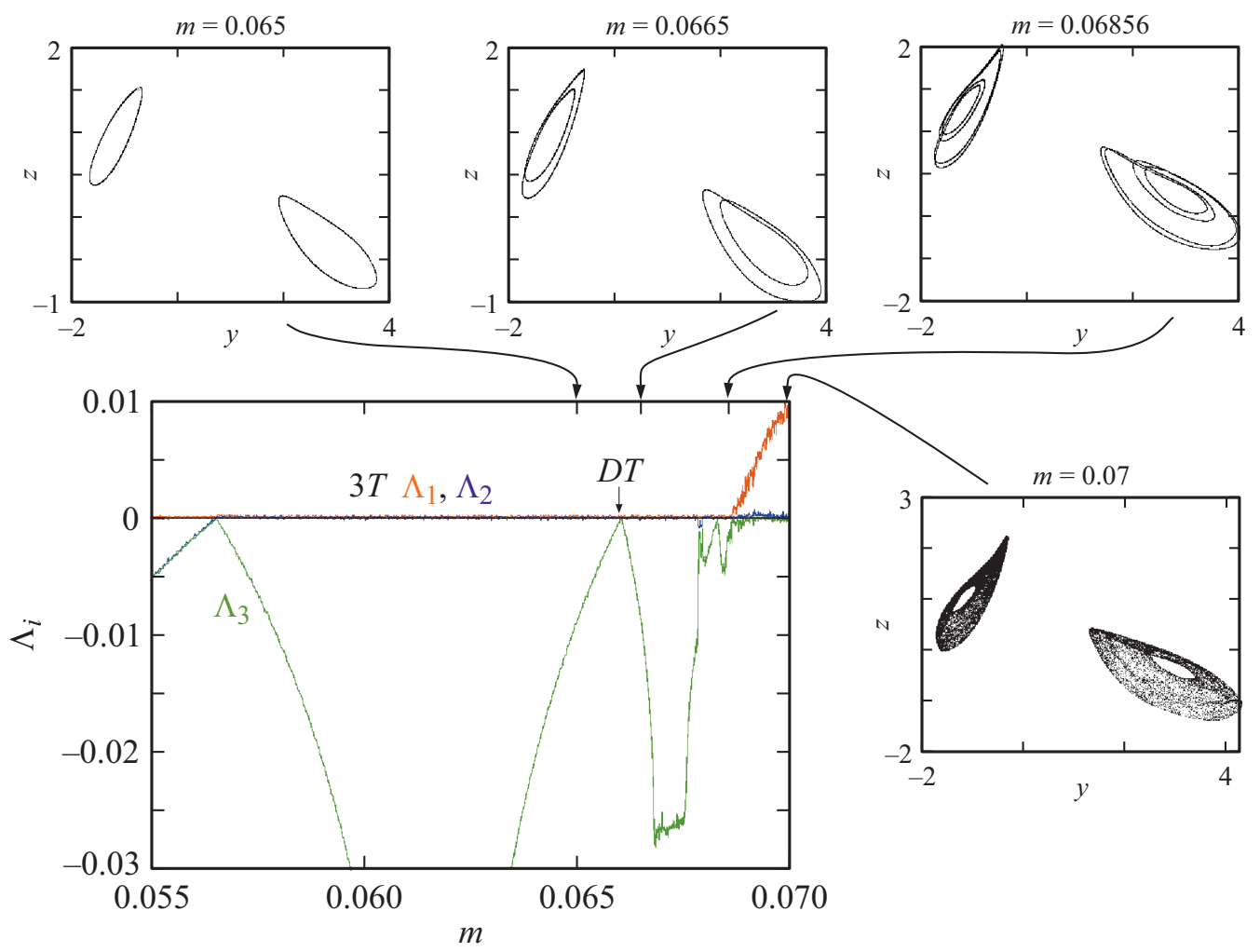

Рис. 1. Портреты в двойном сечении Пуанкаре трехчастотных торов $3 T$ (на вставках) и графики показателей Ляпунова $\Lambda_{i}$ системы (3) в зависимости от параметра возбуждения $m . a=0.03, \omega=4$. $D T$ - точка удвоения трехчастотного тора.

два гладких овала. С ростом $m$ в точке $D T$ происходит удвоение такого тора, а затем наблюдается его разрушение.

На основной части рис. 1 показаны графики трех старших показателей Ляпунова системы (3) в зависимости от параметра возбуждения $m$. Отметим, что для потоковых систем один показатель всегда равен нулю. Мы же вычисляем показатели в стробоскопическом сечении, так что этот нулевой показатель оказывается отброшенным. Таким образом, трехчастотному тору отвечает равенство нулю двух показателей: $\Lambda_{1}=\Lambda_{2}=0$ (аналогичная ситуация имеет место для дискретных отображений [10]). Представленные графики также подтверждают характер бифуркации: в ее точке график показателя $\Lambda_{3}$ обращается в нуль, оставаясь отрицательным в ее окрестности. Это признак бифуркации удвоения тора $[11,12]$.

Рассмотрим теперь, как меняется поведение системы в зависимости от амплитуды воздействия $a$, если при $a=0$ в системе наблюдается хаос. Зафиксируем значение параметра $m=0.07$, когда тор разрушен. Графики показателей Ляпунова в зависимости от амплитуды воздействия $a$ показаны на рис. 2. Можно видеть, что при малых амплитудах, как и следовало ожидать, реализуется хаотический или гиперхаотический режим с одним или двумя положительными показателями Ляпунова. При больших амплитудах, однако, возникает периодический режим $P$, когда все показатели отрицательны. Таким образом, в системе возникает эффект подавления хаоса периодической внешней силой [13]. Два показателя Ляпунова при этом совпадают $\left(\Lambda_{1}=\Lambda_{2}\right)$ и в точке $N S$ обращаются в нуль. Это точка бифуркации Неймарка-Сакера, в результате которой возникает двухчастотный квазипериодический режим $2 T$, когда $\Lambda_{1}=\Lambda_{2}=0$. На вставке к рис. 2 показан соответствующий аттрактор в стробоскопическом сечении, который представляет собой замкнутую инвариантную кривую. Таким образом, в данной системе за счет подавления хаоса возникает не только периодический режим типа описанного в [13], но и квазипериодический режим, который занимает обширную область по амплитуде воздействия. При дальнейшем уменьшении амплитуды воздействия тор удваивается, а затем разрушается.

На рис. 3 приведено бифуркационное дерево для $\omega=6$. Можно видеть точку бифуркации Неймарка-Сакера $N S$ и двухчастотный квазипериодический режим $2 T$.

Таким образом, воздействие гармонического сигнала на квазипериодический генератор Анищенко-Астахова приводит к возможности новых эффектов. При малой амплитуде воздействия это удвоение трехчастотного тора, при большой - эффект подавления хаоса, приводящий не только к периодическому, но и к квазипериодическому режиму. 


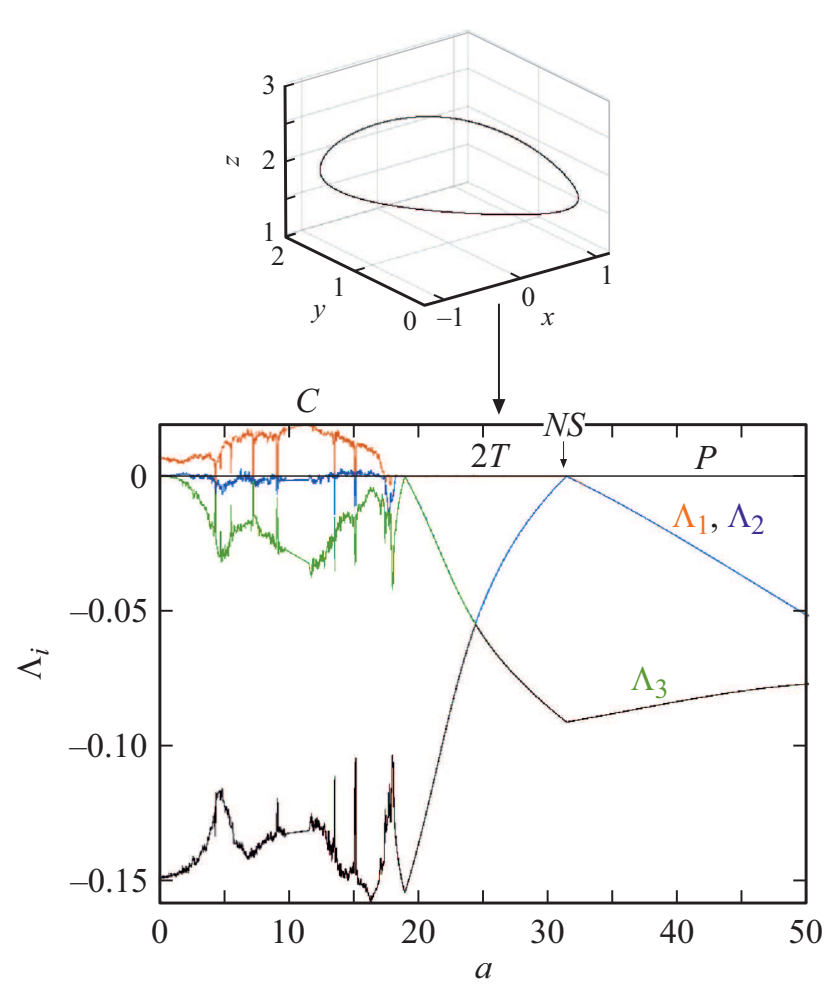

Pис. 2. Портрет в стробоскопическом сечении (на вставке) и графики показателей Ляпунова $\Lambda_{i}$ системы (3). $m=0.07$, $\omega=6$. $P$ - область периодических режимов, $2 T-$ область двухчастотных торов, $C-$ область хаоса, $N S-$ точка бифуркации Неймарка-Сакера.

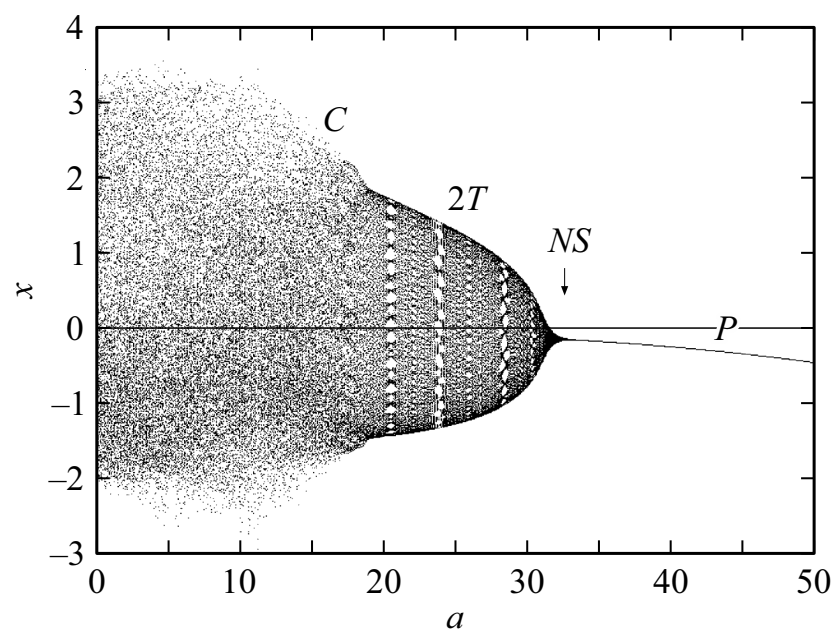

Рис. 3. Бифуркационное дерево системы (3), построенное с использованием стробоскопического отображения. $m=0.07$, $\omega=6$.

\section{Финансирование работы}

Работа поддержана грантом Российского научного фонда № 21-12-00121 (https://rscf.ru/project/21-12$00121 /)$.

\section{Конфликт интересов}

Авторы заявляют, что у них нет конфликта интересов.

\section{Список литературы}

[1] В.С. Анищенко, В.С. Астахов, Т.Е. Вадивасова, Изв. Саратов. ун-та. Новая серия. Сер. Физика, 5 (1), 54 (2005). DOI: $10.18500 / 1817-3020-2005-5-1-54-68$

[2] В.С. Анищенко, Сложные колебания в простых системах. Механизмы возникновения, структура и свойства динамического хаоса в радиофизических системах (URSS, M., 2009).

[3] В.С. Анищенко, Т.Е. Вадивасова, Лекции по нелинейной динамике (НИЦ „Регулярная и хаотическая динамика“, М.-Ижевск, 2011).

[4] В.С. Анищенко, С.М. Николаев, Письма в ЖТФ, 31 (19), 88 (2005). [V.S. Anishchenko, S.M. Nikolaev, Tech. Phys. Lett., 31 (10), 853 (2005). DOI: 10.1134/1.2121837].

[5] V. Anishchenko, S. Nikolaev, J. Kurths, Phys. Rev. E, 73 (5), 056202 (2006). DOI: 10.1103/PhysRevE.73.056202

[6] V. Anishchenko, S. Nikolaev, J. Kurths, Phys. Rev. E, 76 (4), 046216 (2007). DOI: 10.1103/PhysRevE.76.046216

[7] V.S. Anishchenko, S.M. Nikolaev, Int. J. Bifurcat. Chaos, 18 (09), 2733 (2008). DOI: 10.1142/S0218127408021956

[8] N. Stankevich, A. Kuznetsov, E. Popova, E. Seleznev, Nonlinear Dyn., 97 (4), 2355 (2019). DOI: $10.1007 / \mathrm{s} 11071-019-05132-0$

[9] I.R. Sataev, N.V. Stankevich, Chaos, 31 (2), 023140 (2021). DOI: $10.1063 / 5.0038878$

[10] A.P. Kuznetsov, Yu.V. Sedova, Int. J. Bifurcat. Chaos, 24 (07), 1430022 (2014). DOI: 10.1142/S0218127414300225

[11] R. Vitolo, H. Broer, C. Simó, Regul. Chaot. Dyn., 16 (1-2), 154 (2011). DOI: 10.1134/S1560354711010060

[12] А.П. Кузнецов, И.Р. Сатаев, Н.В. Станкевич, Л.В. Тюрюкина, Физика квазипериодических колебаний (Изд. центр „Наука“, Саратов, 2013).

[13] A. Pikovsky, M. Rosenblum, J. Kurths, Synchronization: a universal concept in nonlinear sciences (Cambridge University Press, 2001). 